\title{
TELE-DYSPHAGIA MANAGEMENT: AN OPPORTUNITY FOR PREVENTION, COST-SAVINGS AND ADVANCED TRAINING
}

\author{
JAMES COYLE, PHD, CCC-SLP, BRS-S

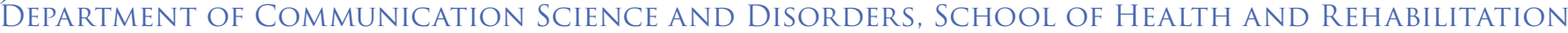 \\ SCIENCES, UniVERSity of PitTSBURGH, PITTSBURGH, PENNSYLVANiA, USA
}

\section{ABSTRACT}

\begin{abstract}
Many patients survive severe stroke because of aggressive management in intensive care units. However, acquiring pneumonia during the post-onset phase significantly reduces both the quality and likelihood of survival. Aspiration pneumonia (AP), a relatively recent addition to the list of the pneumonias, is associated with dysphagia, a swallowing disorder that may cause aspiration of swallowed food or liquids mixed with bacterial pathogens common to saliva, or by aspiration of gastric contents due to emesis or gastroesophageal reflux. While it is within the purview of speech-language pathologists to provide evaluation, treatment, and management of dysphagia, the number of patients with dysphagia is growing faster than the number of qualified dysphagia clinicians. Because dysphagia consultations via telepractice are feasible and relatively accessible from a technological standpoint, they offer a promising strategy to bring the expertise of distant dysphagia experts to patients in underserved areas. Tele-dysphagia management has the potential to increase patients' survival, enhance the expertise of primary, local clinicians, and reduce healthcare costs. Even a modest reduction in either hospital admissions for aspiration pneumonia, or in the length of stay for AP, could save the US health care system hundreds of millions of dollars each year. Wide spread tele-dysphagia management offers significant opportunities for prevention, cost-savings and advanced training, and is therefore worthy of consideration by stakeholders in the health care system and university training programs.
\end{abstract}

Keywords: Aspiration pneumonia, dysphagia, speech-language pathology, tele-dysphagia, telepractice

\section{PNEUMONIA AND DYSPHAGIA:}

\section{A LETHAL COMBINATION}

While many patients survive severe stroke because of aggressive management in intensive care units, acquiring pneumonia during the post-onset phase significantly reduces both the quality and likelihood of survival. Conditions such as stroke that cause temporary or permanent changes in sensorimotor function often compromise airway protection during swallowing.

In elderly patients with stroke, swallowing disorders (i.e., dysphagia) increase mortality. Hilker et al. (2003) prospectively compiled data from the medical records of stroke patients admitted to a neurological intensive care unit. Twenty-one percent of their cohort acquired strokeassociated pneumonia (SAP). Within the SAP group, mortality was three times more likely (both short-term and long-term), and these patients had significantly less rehabilitative success compared to stroke patients without SAP (Hilker et al., 2003). In a large, population-based study of 11,286 stroke patients without co-occurring terminal disease or advance directives requiring "do not resuscitate" management, the mortality rate was six times higher (26.9\%) in patients who developed pneumonia after onset of stroke compared to those that did not develop pneumonia (4.4\%) (Katzan, Cebul, Husak, Dawson, \& Baker, 2003). After adjustments for various underlying factors affecting mortality, the relative risk of death (RRD) of stroke patients who developed pneumonia within 30 days post-onset was 2.99 (i.e., the probability of death was about three times higher) compared to stroke patients without pneumonia. 


\section{ASPIRATION PNEUMONIA AND DYSPHAGIA}

Aspiration pneumonia (AP), a relatively recent addition to the list of the pneumonias ${ }^{1}$, is associated with dysphagia, a swallowing disorder that may cause aspiration of swallowed food or liquids mixed with bacterial pathogens that are colonizing saliva, or by aspiration of gastric contents due to emesis or gastroesophageal reflux. The assessment of oropharyngeal swallowing function by a qualified clinician is one tactic through which evidence that dysphagia is a likely cause of a patient's pneumonia, - can be distinguished from signs of other sources of pneumonia. This assessment involves a clinical (bedside) examination and in many cases, instrumental assessment using videofluoroscopy or fiberoptic instrumentation.

The incidence of AP has increased dramatically in recent years. In fact, Baine, Yu, \& Summe, (2001) reported that diagnoses of AP had reached epidemic proportions according to an epidemiological study which examined prevalence data published in the American Journal of Public Health. Baine and colleagues reviewed $5 \%$ of all Medicare hospital inpatient bills from 1991 through 1998 to determine the estimated number of hospitalizations for AP in the United States. Their 5\% sample revealed that the frequency of hospitalization for all categories of pneumonia grew from 30,292 to 37,153 during that seven year period (a 22\% increase). Of those admissions, the rate of AP admissions nearly doubled (i.e., from 2974 to 5756 patients) and ranked second only to 'unspecified causes of pneumonia' as coded in hospital discharge summaries (Baine, Yu, \& Summe, 2001). Extrapolating these data to the population, approximately $15.5 \%$ of Medicare hospital admissions for pneumonia in 1998 were classified as aspiration pneumonia. AP carried the highest case-fatality rate during hospitalization (23.1\%) of all pneumonia diagnoses.

\section{ECONOMIC IMPACT OF ASPIRATION PNEUMONIA}

In an analysis of Medicare source data, Niederman, McCombs, Unger, Kumar, and Popovian (1998) determined that more than $\$ 8$ billion was spent treating hospital inpatients for CAP, at an average cost per stay of six to seven thousand dollars. Combining information from both of the aforementioned Medicare review studies, the proportion of CAP inpatient hospital cost for AP was $15.5 \%$ of $\$ 8.4$ billion, or approximately $\$ 1.3$ billion (in 1998 dollars).

Given these figures, the impact of a modest reduction in either hospital admissions for aspiration pneumonia, or in the length of stay for AP, could save the health care system hundreds of millions of dollars each year.

\section{Dysphagia Clinician SHORTAGES}

The number of patients with dysphagia is growing rapidly as the population ages and as life preserving technology prevents death from previously fatal diseases. However the number of qualified dysphagia clinicians is not keeping pace with the increasing incidence of dysphagia. While many urban areas and academically oriented medical institutions may have one or more expert dysphagia clinicians among their resources, rural and other underserved geographic areas are often dependent on the skills of well-intentioned, hardworking clinicians with few opportunities to specialize in the management of swallowing disorders.

Clinicians providing services in some areas need to have a broad base of clinical skills to be prepared for a diverse caseload. They often work alone or within a very small staff of clinicians responsible for managing speech, language, cognitive, swallowing and feeding disorders in their geographic area. Since they need to be at least minimally competent in all of these areas and do not have large caseloads of patients with similar disorders, they are unable to focus narrowly on a specific area of clinical practice to develop expertise. Just as a rural physician would refer a patient with an obscure presentation to a specialist in an academic setting, speech-language pathologists often need consultation with expert colleagues specializing in dysphagia assessment and management.

1. Community acquired pneumonia (CAP) is can be caused by microorganisms that are able to thrive in respiratory epithelium. Among bacterial causes of CAP, pneumococcus and streptococcus pneumoniae are the most common, and result in 50,000 deaths annually (Centers for Disease Control and Prevention, 2009). Other forms of bacterial pneumonia include: (a) nosocomial pneumonia, caused by pathogens typical in hospitals and nursing homes, (b) ventilator-associated pneumonia, caused by contamination of mechanical ventilator circuits, and (c) mycoplasma pneumonia. Viral forms of pneumonia include Legionella and respiratory syncytial virus. 
Unlike these generalists who strive to do the best they can under the conditions of their work environment, experts have had the opportunity to undergo the type of rigorous training and advanced postgraduate education needed to accurately sort through the numerous and often subtle signs and symptoms that lead them to an accurate diagnosis and treatment plan. The Specialty Board on Swallowing and Swallowing Disorders, in concert with the American-Speech-Language-Hearing Association, grants the designation of BRS-S or Board Recognized Specialist in Swallowing and Swallowing Disorders to speech-language pathologists who have demonstrated their expertise in dysphagia.

\section{TELE-DYSPHAGIA OPPORTUNITIES}

Perlman and Witthawaskul (2002) and Malandraki, McCullough, He, McWeeny , and Perlman (2011) have demonstrated that remote dysphagia consultations are feasible and relatively accessible from a technological standpoint. Telepractice therefore presents opportunities to link the expertise of qualified dysphagia experts to underserved areas.

A second advantage of the telepractice consultation in dysphagia management is the opportunity to not only provide a higher quality of patient care through the collaboration between the primary clinician and remote expert, but to provide by example and consultation, advanced training to the primary clinician through the conduct of the case management by the expert.

\section{LOOKING FORWARD}

Dysphagia consultations via telepractice present opportunities for millions of dollars in annual costsavings. Given the drive to reduce US health care costs, it is a propitious time to shine a light on this new service delivery model. Potential stakeholders include: consumers, clinicians, insurers, health care systems, university training programs, providers of post-graduate continuing education, professional associations and specialty boards.

While dysphagia consultations via telepractice are feasible and relatively accessible from a technological standpoint, telepractice clinical protocols require development and strategic deployment. Tele-dysphagia management must be well informed by empirical data that demonstrate efficacy, economic savings, and work-force enhancement.

\section{REFERENCES}

1. Baine, W.B., Yu, W., \& Summe, J.P. (2001). Epidemiologic trends in the hospitalization of elderly Medicare patients for pneumonia, 1991-1998. American Journal of Public Health,91,1121-1123.

2. Centers for Disease Control and Prevention, (2009), Retrieved from: http://www.cdc.gov/nchs/data/dvs/ deaths_2009_release.pdf

3. Hilker, R., Poetter, C., Findeisen, N., Sobesky, J., Jacobs, A., Neveling, M., \& Heiss, W.D. (2003). Nosocomial pneumonia after acute stroke: Implications for neurological intensive care medicine. Stroke, 34,975-81.

4. Katzan, I.L., Cebul, R.D., Husak, S.H., Dawson, N.V., \& Baker, D.W. (2003). The effect of pneumonia on mortality among patients hospitalized for acute stroke. Neurology, 60,620-625.

5. Malandraki, G.A., McCullough, G., He, X., McWeeny, E., \& Perlman, A.L. (2011). Teledynamic evaluation of oropharyngeal swallowing. Journal of Speech, Language, and Hearing Research, 54,1497-505

6. Niederman, M.S., McCombs, J.S., Unger, A.N., Kumar, A., \& Popovian, R. (1998). The cost of treating communityacquired pneumonia. Clinical Therapeutics, 20,820-837.

7. Perlman, A. L., \& Witthawaskul, W. (2002). Real-time remote telefluoroscopic assessment of patients with dysphagia. Dysphagia, 17, 162-167. 
